Bond University

Research Repository

\title{
Strongman vs. traditional resistance training effects on muscular function and performance
}

Winwood, Paul W; Cronin, John B; Posthumus, Logan R; Finlayson, Steven J; Gill, Nicholas D; Keogh, Justin W L

Published in:

Journal of Strength and Conditioning Research

DOI:

10.1519/JSC.0000000000000629

Licence:

Other

Link to output in Bond University research repository.

Recommended citation(APA):

Winwood, P. W., Cronin, J. B., Posthumus, L. R., Finlayson, S. J., Gill, N. D., \& Keogh, J. W. L. (2015).

Strongman vs. traditional resistance training effects on muscular function and performance. Journal of Strength and Conditioning Research, 29(2), 429-439. https://doi.org/10.1519/JSC.0000000000000629

\footnotetext{
General rights

Copyright and moral rights for the publications made accessible in the public portal are retained by the authors and/or other copyright owners and it is a condition of accessing publications that users recognise and abide by the legal requirements associated with these rights.
}

For more information, or if you believe that this document breaches copyright, please contact the Bond University research repository coordinator. 
STRONGMAN VERSUS TRADITIONAL RESISTANCE TRAINING EFFECTS ON MUSCULAR FUNCTION AND PERFORMANCE

"This manuscript contains material that is original and not previously published in text or on the Internet, nor is it being considered elsewhere until a decision is made as to its acceptability by the "Journal of Strength and Conditioning Research"

Paul W Winwood

Bay of Plenty Polytechnic

Windermere Drive

Tauranga 3143

Ph: $08002677659 \times 6125$

Email: paul.winwood@boppoly.ac.nz 
Title: Strongman versus traditional resistance training effects on muscular function and performance

Running head: Strongman Training Effects

Key Words: weight training, functional, transference, variation

Authors:

Winwood, P. W ${ }^{1,2}$., Cronin, J. B ${ }^{1,3}$., Posthumus, L. R ${ }^{1,2}$., Finlayson, S. ${ }^{1,2}$., Gill, N. D ${ }^{1}$ \& Keogh, J. W. L ${ }^{1,4,5}$.

${ }^{1}$ Sports Performance Research Institute New Zealand (SPRINZ)

AUT Millennium Institute

AUT University, Auckland

New Zealand

${ }^{2}$ Bay of Plenty Polytechnic

Department of Sport and Recreation

School of Applied Science

Tauranga

New Zealand

${ }^{3}$ Edith Cowan University

School of Exercise, Biomedical and Health Sciences

Perth

Australia 
${ }^{4}$ Faculty of Health Sciences and Medicine

Gold Coast

Australia

${ }^{5}$ University of the Sunshine Coast

Cluster for Health Improvement, Faculty of Science, Health, Education and Engineering

Queensland

Australia

Corresponding author:

Paul Winwood

Department of Sport and Recreation

School of Applied Science

Bay of Plenty Polytechnic, Private Bag 12001

Tauranga 3143

Ph 08002677659 x6125 Email paul.winwood@boppoly.ac.nz 


\section{$\underline{\text { Abstract }}$}

Currently, no evidence exists as to the effectiveness of strongman training programs for performance enhancement. This study compared the effects of seven weeks of strongman resistance training versus traditional resistance training on body composition, strength, power, and speed measures. Thirty experienced resistance-trained rugby players were randomly assigned to one of two groups; strongman $(n=15$; mean $\pm \mathrm{SD}$ : age, $23.4 \pm 5.6$ years; body mass, $91.2 \pm 14.8 \mathrm{~kg}$; height, $180.1 \pm 6.8 \mathrm{~cm})$ or traditional $(\mathrm{n}=15$; mean $\pm \mathrm{SD}$ : age, $22.5 \pm 3.4$ years; body mass, $93.7 \pm 12.3 \mathrm{~kg}$; height, $181.3 \pm 5.9 \mathrm{~cm})$. The strongman and traditional training programs required the participants to train twice a week and contained exercises that were matched for biomechanical similarity with equal loading. Participants were assessed for body composition, strength, power, speed and change of direction (COD) performance. Within-group analyses indicated that all performance measures improved with training $(0.2 \%$ to $7 \%)$ in both the strongman and traditional training groups. No significant between-group differences were observed in functional performance measures after 7-weeks of resistance training. Between group differences indicated small positive effects in muscle mass and acceleration performance and large improvements in 1RM bent over row strength associated with strongman compared to traditional training. Small to moderate positive changes in 1RM squat and deadlift strength, horizontal jump, COD turning ability and sled push performance were associated with traditional compared to strongman training. Practitioners now have the first evidence on the efficacy of a strongman training program and it would seem that short term strongman training programs are as effective as traditional resistance training programs in improving aspects of body composition, muscular function and performance. 


\section{$\underline{\text { Introduction }}$}

In recent years, the use of strongman training modalities for performance enhancement have become popular in strength and conditioning practice $(4,10,15,16,42,46)$. This increase in popularity could be attributed to the unique events demonstrated in the sport, the increasing accessibility of the training implements and the opportunity to use these exercises to add variation to resistance training programs. Generally, gymnasium-based resistance training exercises are performed vertically with two feet side by side. While walking lunges or split stance exercises may offset some of the limitations of the traditional lifts (20), strongman exercises such as the farmers walk and heavy sled pull may be even more applicable to sporting movements as they often involve unstable and awkward resistances and involve both unilateral and bilateral motion. Stone and colleagues (33) have suggested that the more similar a training exercise is to actual physical performance, the greater the probability of transfer. Advocates of strongman training $(2,10,16,30,38,46)$ have suggested it is more specific than other forms of strength training and may help 'bridge' the gap between gymnasium-based strength training and functional performance. A recent study of 220 strength and conditioning coaches found that $81 \%$ believe they had achieved good to excellent results from strongman implement training (42). Such a contention however, is speculative given that no research to the knowledge of these authors has examined the chronic effects of strongman training compared to typical gymnasium-based strength training of athletes.

Articles published on the sport of strongman have provided valuable insight into how strongman implement training may be implemented in strength and conditioning programs (4, 16, 38, 46). Researchers have investigated the metabolic and endocrine responses, and biomechanical (kinematic determinants of performance and lower back/hip loads) demands 
of strongman exercises $(5,12,21,22,26)$. These cross-sectional studies have provided results suggesting that strongman events could prove useful in improving core strength, power, sprint start and acceleration capabilities, as well as anaerobic conditioning and for increasing energy expenditure. However, an evidence-based approach that uses longitudinal designs to determine the efficacy of strongman training is needed before strength and conditioners find reason to change current training strategies and best practice.

In light of the limitations of the literature reviewed and given that no study has investigated the effectiveness of a strongman resistance program, the purpose of this study was to compare the chronic effects of strongman implement training versus traditional training on aspects of muscular function and performance. Such a comparison should improve our understanding of the effects of strongman exercises and how they may differ to that of traditional type gymnasium-based approach. It was hypothesised (based on the principle of specificity) that at the end of the training intervention, effect sizes in grip strength and horizontal performance tests e.g. sprinting speed, change of direction (COD) time, medicine ball throw and horizontal jump distance would be greater in the strongman training group, whereas effect sizes in the vertical performance measures including vertical jump height and 1RM strength would be greater in the traditional training group.

\section{$\underline{\text { Methods }}$}

\section{Experimental Approach to the Problem}

A randomised controlled trial was used to compare a traditional resistance and strongman training protocol. Thirty experienced resistance-trained rugby players volunteered to participate in this study. Participants were assessed for body composition, $30 \mathrm{~m}$ sprint time, horizontal jump distance, seated medicine ball chest press throw, vertical jump height, grip 
strength, $15 \mathrm{~m}$ sled push and 5-0-5 change of direction tests (respectively). Baseline testing occurred in week one, after which a supervised seven week strength and power programme was performed twice weekly before final testing in week nine. Changes in the outcome variables after training were compared between groups using independent T-tests and effect statistics.

\section{Participants}

Thirty male resistance-trained amateur and semi-professional rugby players volunteered to participate in this study. A summary of the participants characteristics are presented in Table 1. All participants regularly performed resistance training as part of their training and had a strength training background ( $>1$ year). The study was conducted in the participant's offseason where the majority of participants were at the start of a training cycle aimed at improving their strength performance. Participants were excluded if: any medical problems were reported that compromised their participation or performance in this study; and, athletes were taking or had previously taken any performance-enhancement drugs of any kind. All participants provided written informed consent after having being briefed on the potential risks associated with this research. Prior ethical approval was granted by the AUT University Ethics Committee, Auckland, New Zealand. In total, 36 participants were recruited for this study, but, because of injury, transport issues and work and family commitments, only 30 participants completed all parts of the testing and intervention program. The results of this study are based on the data obtained from these 30 participants. Two injuries were reported as part of the training intervention. One was a minor back muscle sprain associated with the deadlift, which resulted in the participant missing one training session and the other was a shoulder injury associated with strongman training in which the participant had to stop training and subsequently pull out of the study. Adherence to training was $98.6 \%$ for both 
groups. All training for this study was undertaken at a similar time of day with participants instructed to maintain their normal dietary intake before and after each workout. We did not control for nutrition, or hydration levels but participants were told not to make any changes in the above during the intervention and post intervention testing.

Table 1: Participant characteristics (mean \pm standard deviation)

\begin{tabular}{llll}
\hline Parameters & $\begin{array}{l}\text { All Participants } \\
(\mathbf{n}=\mathbf{3 0})\end{array}$ & $\begin{array}{l}\text { Strongman } \\
\text { Group } \\
(\mathbf{n}=\mathbf{1 5})\end{array}$ & $\begin{array}{l}\text { Traditional Group } \\
(\mathbf{n}=\mathbf{1 5})\end{array}$ \\
\hline Age (y) & $22.9 \pm 4.6$ & $23.4 \pm 5.6$ & $22.5 \pm 3.4$ \\
Height (cm) & $180.7 \pm 6.2$ & $180.1 \pm 6.8$ & $181.3 \pm 5.9$ \\
Body mass (kg) & $92.5 \pm 13.4$ & $91.2 \pm 14.8$ & $93.7 \pm 12.3$ \\
$\begin{array}{l}\text { Resistance training experience } \\
(y)\end{array}$ & $4.3 \pm 2.8$ & $3.9 \pm 2.3$ & $4.7 \pm 3.3$ \\
\hline
\end{tabular}

IRM Strength Measures

$\begin{array}{llll}\text { Clean and jerk }(\mathrm{kg}) & 85.1 \pm 11.8 & 87.2 \pm 9.3 & 81.5 \pm 14.4 \\ \text { Deadlift }(\mathrm{kg}) & 171.1 \pm 23.7 & 181.3 \pm 18.2 & 161.8 \pm 24.1 \\ \text { Military press }(\mathrm{kg}) & 69.1 \pm 11.4 & 68.5 \pm 10.6 & 69.6 \pm 12.1 \\ \text { Squat }(\mathrm{kg}) & 142.4 \pm 25.0 & 141.1 \pm 24.0 & 146.2 \pm 24.0 \\ \text { Bent over row }(\mathrm{kg}) & 106.0 \pm 14.2 & 106.9 \pm 14.6 & 108.2 \pm 11.9\end{array}$

Performance Measures

$30 \mathrm{~m}$ sprint speed (s)

$4.36 \pm 0.20$

$4.35 \pm 0.20$

$4.38 \pm 0.20$

505 COD test (s)

$2.39 \pm 0.12$

$2.40 \pm 0.13$

$2.38 \pm 0.12$

$15 \mathrm{~m} 70 \mathrm{~kg}$ sled push (s)

$4.03 \pm 0.33$

$4.01 \pm 0.37$

$4.06 \pm 0.30$

Vertical jump (cm)

$58.28 \pm 8.80$

$59.87 \pm 9.52$

$56.57 \pm 7.94$

Horizontal jump (m)

$2.38 \pm 0.18$

$2.40 \pm 0.21$

$2.35 \pm 0.16$

$5 \mathrm{~kg}$ MB Chest throw (m)

$4.65 \pm 0.54$

$4.56 \pm 0.52$

$4.76 \pm 0.56$

Grip strength left $(\mathrm{kg})$

$55.68 \pm 7.85$

$56.00 \pm 7.34$

$55.36 \pm 8.57$

Grip strength right (kg)

$56.20 \pm 8.64$

$56.33 \pm 9.66$

$56.07 \pm 7.86$ 


\section{$\underline{\text { Strength Testing }}$}

No supportive aids beyond the use of a weightlifting belt and lifting chalk were permitted during the testing. The warm up, loading increments and rest periods used were according to previously established protocols (40). Movement competency screening of the 1RM strength exercises took place prior to strength testing and instruction was given when required to improve technique. Strength testing was assessed by 1RM - 3RM tests performed with a freeweight Olympic-style barbell. The 1RM test was performed for the clean and jerk and 1RM 3RM tests were performed for deadlift, military press, squat, and bent over row (respectively). Squat RM was assessed using the methods outlined by Baker (3). Completed lifts in the clean and jerk, deadlift and military press were recognised when the participants were standing still and fully upright with the applied load. For the bent over row, participants had to achieve full range of motion of the upper limbs while remaining in a partial squat position with no movement at the hip and knee. The Poliquin formula (29) was used to determine the participants predicted $1 \mathrm{RM}$ from their $2 \mathrm{RM}$ or $3 \mathrm{RM}$ values. Percentage of loading for the training intervention was based on the athletes predicted 1RM.

\section{Functional Performance Testing}

Before the commencement of functional performance testing participants had their body composition (body mass, body fat percentage and muscle mass (MM), measured and recorded using a bioelectrical impedance machine (InBody230, Biospace). Participants then performed a ten minute standardised warm up prior to testing that consisted of of dynamic stretching, and light jogging interspersed with bodyweight exercises. Testing commenced five minutes after the warm up. The testing session involved the determination of the participants $5 \mathrm{~m}, 15 \mathrm{~m}$ and $30 \mathrm{~m}$ sprint times (s) from a $30 \mathrm{~m}$ sprint, horizontal jump (m), seated $5 \mathrm{~kg}$ medicine ball (MB) chest press throw (m), vertical jump height $(\mathrm{cm})$, left and 
right hand grip strength $(\mathrm{kg}), 70 \mathrm{~kg} 15 \mathrm{~m}$ sled pushes (s), and 5-0-5 change of direction (COD) test (s). A rest period with a minimum of 10-minutes was provided between each test. Participants performed two; $30 \mathrm{~m}$ sprints $(\mathrm{CV}=0.6 \%), 5-0-5 \mathrm{COD}$ tests $(\mathrm{CV}=2.2 \%)$, grip strength tests $(\mathrm{CV}=4.2 \%$ and $4.5 \%$ for left and right grip strength, respectively), and $15 \mathrm{~m}$ sled pushes $(\mathrm{CV}=2.9 \%)$; and three horizontal jumps $(\mathrm{CV}=1.6 \%)$, countermovement vertical jumps $(\mathrm{CV}=3.2 \%)$ and seated $5 \mathrm{~kg} \mathrm{MB}$ chest press throws $(\mathrm{CV}=1.3 \%)$. The best result for each test was used for data analysis. All pre-and-post functional performance testing were performed indoors on artificial turf $(15 \mathrm{~mm}$ underlay/10 $\mathrm{mm}$ overlay) at the same time of day. The performance tests chosen for this study have been considered appropriate functional performance tests and conditioning exercises for a variety of athletes and have shown good test-retest reliability $(11,13,25,27,39)$.

\section{Strength and Power Assessment}

Grip strength was determined with a grip strength dynamometer (TTM Original Dynamometer 100kg, Tokyo). Participants were instructed to hold the dynamometer at their side and pull the handles together with maximal effort for up to three seconds. The countermovement vertical jump (CVJ) and horizontal jump were performed off two feet and with full arm motion. A tape measure was used to determine horizontal jump distance and the Vertec Yardstick (Swift Performance Equipment, Australia) was used to determine jump height. Standing reach measures were subtracted from the Vertec determined jump height to calculate the CVJ displacement. Horizontal jump was measured from the start line (positioned in front of toes) to the nearest point of contact on landing (back of the heel). Participants were required to jump as far forward as possible and land on two feet without falling. Participants were allowed two familiarization horizontal jumps and were instructed to 'sink' into the landing to prevent falling forward. 


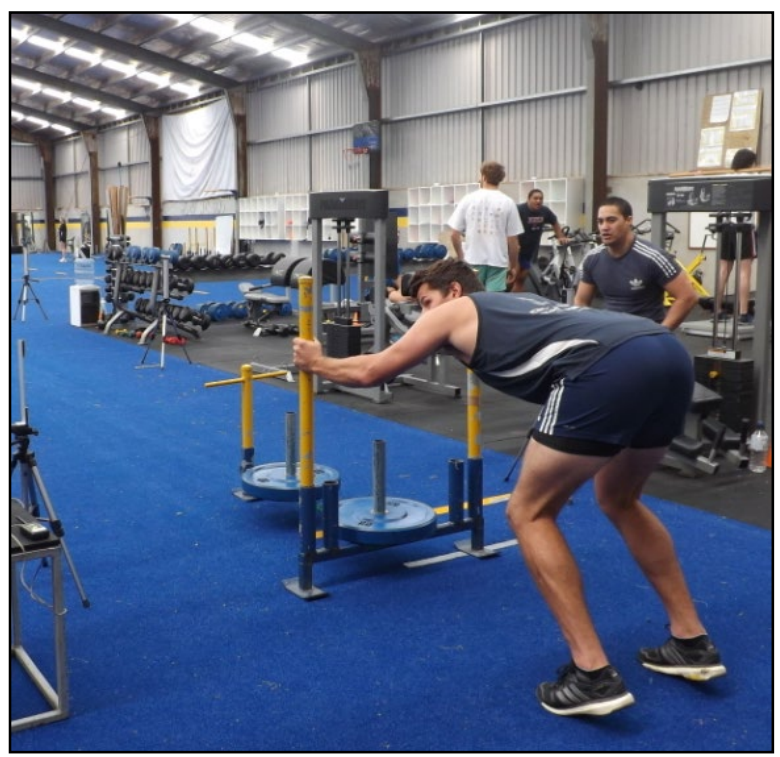

Figure 1: $70 \mathrm{~kg} 15 \mathrm{~m}$ heavy prowler push

The $70 \mathrm{~kg}$ sled push over $15 \mathrm{~m}$ (see Figure 1) was measured using SpeedlightV2 wireless dual beam timing lights (Swift Performance Equipment, Australia). Participants started in a bilateral standing stance with the sled poles positioned $0.5 \mathrm{~m}$ before the start line. No rocking or backward steps were allowed prior to the start. Participants were instructed to push the sled as fast as possible with maximal effort. Hand pushing position was determined by the first web space with participants standing anteriorly to the sled poles with straight arms at their sides. Timing lights were placed at the start, $5 \mathrm{~m}, 10 \mathrm{~m}$ and $15 \mathrm{~m}$ marks. Timing light beams were set at $92.5 \mathrm{~cm}$ (top beam) and $68 \mathrm{~cm}$ (bottom beam) for all performance test times represented in this study. Push sled times were recorded for total distance and between each split. The 5-kg seated concentric MB chest press throw was performed with the participant sitting on the floor with legs fully extended, approximately $60 \mathrm{~cm}$ apart and the back and head against a wall. The ball was held with the hands on the side and against the centre of the chest with the forearms positioned parallel to the ground. Participants were instructed to throw the medicine ball explosively at a 45 degree angle to the horizontal as far as possible 
while keeping the head and back against the wall. Participants were instructed to throw the MB along a line in which a measuring tape was adhered too. The distance of ball flight was recorded.

\section{Speed and Change of Direction Assessment}

Speed and 5-0-5 COD ability were measured using SpeedlightV2 wireless dual beam timing lights (Swift Performance Equipment, Australia). For both tests, participants started in a standing split stance, with the toes of the back foot in line with the heel of the front foot, 50 $\mathrm{cm}$ before the start line. No rocking or backward steps were allowed prior to the start. Participants were instructed to sprint at maximal effort in the speed and 505 COD tests. For the $30 \mathrm{~m}$ sprint test, timing lights were placed at the start, $5 \mathrm{~m}, 15 \mathrm{~m}$ and $30 \mathrm{~m}$ marks. Sprint times were recorded for total distance and between each split. For the 5-0-5 COD test, timing lights were placed on the $2 \mathrm{~m}$ and $5 \mathrm{~m}$ markers and times were recorded when the participant passed through the $5 \mathrm{~m}$ and $2 \mathrm{~m}$ markers, turned on the line, and returned through the $2 \mathrm{~m}$ and $5 \mathrm{~m}$ markers. 5-0-5 times were recorded for total distance $(10 \mathrm{~m})$ and between each split $(0-$ $3 \mathrm{~m}$ (deceleration), $2 \mathrm{~m}+2 \mathrm{~m}$ (turning ability), and $2 \mathrm{~m}$ to $5 \mathrm{~m}$ (acceleration)).

\section{$\underline{\text { Training Programs }}$}

The seven week training intervention involved participants performing either traditional resistance training or a strongman training program (Table 2). The traditional and strongman exercises were paired based on biomechanical similarity and loads were equated between the two groups. The exercises chosen are commonly performed in strength and conditioning practice, and by strongman athletes for the development of muscular strength and power (44). 
Table 2: Outline of traditional and strongman training protocols

\begin{tabular}{|c|c|c|c|c|c|}
\hline Protocols & Sets & $\begin{array}{l}\text { Reps or } \\
\text { Distance }\end{array}$ & Total Load & Rest & $\begin{array}{l}\text { Rest Between } \\
\text { Exercises }\end{array}$ \\
\hline \multicolumn{6}{|l|}{ Traditional Protocol } \\
\hline * Clean and Jerk & 3 & 5 reps & $70 \%$ of $1 \mathrm{RM}$ & $2 \mathrm{~min}$ & $3 \mathrm{~min}$ \\
\hline Deadlift & 3 & 5 reps & $80 \%$ of $1 \mathrm{RM}$ & $2 \min$ & $3 \mathrm{~min}$ \\
\hline Military Press & 3 & 6 reps & $80 \%$ of $1 \mathrm{RM}$ & $2 \mathrm{~min}$ & $3 \mathrm{~min}$ \\
\hline *Back Squat & 3 & 5 reps & $85 \%$ of $1 \mathrm{RM}$ & $2 \mathrm{~min}$ & $3 \mathrm{~min}$ \\
\hline One Arm Row & 2 & 8 reps & $\begin{array}{c}30 \% \text { of } 1 \mathrm{RM} \text { Bent } \\
\text { over row }\end{array}$ & $2 \mathrm{~min}$ & \\
\hline \multicolumn{6}{|l|}{ Strongman Protocol } \\
\hline * Log Lift & 3 & 5 reps & $\begin{array}{c}70 \% \text { of } 1 \mathrm{RM} \\
\text { Clean and Jerk }\end{array}$ & $2 \mathrm{~min}$ & $3 \mathrm{~min}$ \\
\hline Farmers Walk & 3 & $28 \mathrm{~m}$ & $\begin{array}{c}80 \% \text { of } 1 \mathrm{RM} \\
\text { Deadlift }\end{array}$ & $2 \min$ & $3 \mathrm{~min}$ \\
\hline Axle Press & 3 & 6 reps & $\begin{array}{l}80 \% \text { of } 1 \mathrm{RM} \\
\text { Military Press }\end{array}$ & $2 \mathrm{~min}$ & $3 \mathrm{~min}$ \\
\hline *Heavy Sled Pull & 3 & $25 \mathrm{~m}$ & $\begin{array}{c}85 \% \text { of } 1 \mathrm{RM} \\
\text { Back Squat }\end{array}$ & $2 \mathrm{~min}$ & $3 \mathrm{~min}$ \\
\hline $\begin{array}{l}\text { Arm Over Arm } \\
\text { Prowler Pull }\end{array}$ & 2 & $\begin{array}{c}16 \text { reps } \\
\text { (8-each arm) }\end{array}$ & $\begin{array}{l}100 \% \text { of } 1 \mathrm{RM} \\
\text { Bent over row }\end{array}$ & $2 \mathrm{~min}$ & \\
\hline
\end{tabular}

Key: *Perform the exercise explosively, $1 \mathrm{RM}=$ One repetition maximum.

Equal training loads $(\mathrm{kg})$ were used for the log lift and clean and jerk, and the axle press and military press. Loading for the arm over arm prowler pull and one arm row was based on the athletes' perceived rate of exertion (Borg's Scale) during pilot studies, and expressed as a \% of 1RM bent over row. For the sled pull and squat, and deadlift and farmers walk loading was equated based on the kinetic data (24). A technical note detailing equations based on time under tension is presented in Appendix 1. Participants were asked to self-select their movement speed for the farmers walk, deadlift and one arm row but were asked to perform the squat, clean and jerk, log press and heavy sled pull as explosively as possible. 
Participants in the strongman group performing the heavy sled pull were instructed to start in a four-point power position and accelerate the sled $25 \mathrm{~m}$ over the artificial turf surface as quickly as possible using powerful triple extension of the lower body. For the arm over arm prowler pull (Prowler sled $30 \mathrm{~kg}, 1400 \mathrm{~mm}$ length, $925 \mathrm{~mm}$ width) participants were instructed to start in a crouching position and pull the rope $(20.0 \mathrm{~kg}$, length $30 \mathrm{~m}, 32 \mathrm{~mm}$ diameter) (Sports Distributors, Tauranga) to the hip with one arm and allow the prowler sled to remain stationary between each pull. For the farmers walk participants were instructed to pick up the bars in each hand and walk forward over a course of $28 \mathrm{~m}$ with the rounding of a cone at half way $(14 \mathrm{~m})$. Participants could choose any technique they wished for the log lift providing that, for a repetition to be counted it had to start from the floor and the participants had to be standing upright with knees and elbows extended. The lifts were performed in a consecutive order (log lift, farmers walk, axle press, heavy sled pull and arm over arm prowler pull). A longer rest period of up to 5-minutes was made available between sets and exercises in both protocols if the participant felt fatigued. Consistent verbal encouragement was provided during testing sessions with the participants frequently reminded to perform specific lifts as fast as possible. The farmers bars $(14.3 \mathrm{~kg}$, length $1160 \mathrm{~mm}$, handle thickness of $33 \mathrm{~mm}$ diameter), axle (17.0 kg, length $2150 \mathrm{~mm}$, diameter 2 inches $)$, sled (11.5 kg, length $600 \mathrm{~mm}$, width $400 \mathrm{~mm})$ and $\log (58.1 \mathrm{~kg}$, length $2355 \mathrm{~mm}$, diameter $165 \mathrm{~mm}$, handle thickness of $33 \mathrm{~mm}$ diameter) used in this study were purchased from Getstrength, Auckland. Pictorial representations of the strongman exercises are presented in Figure 2. 


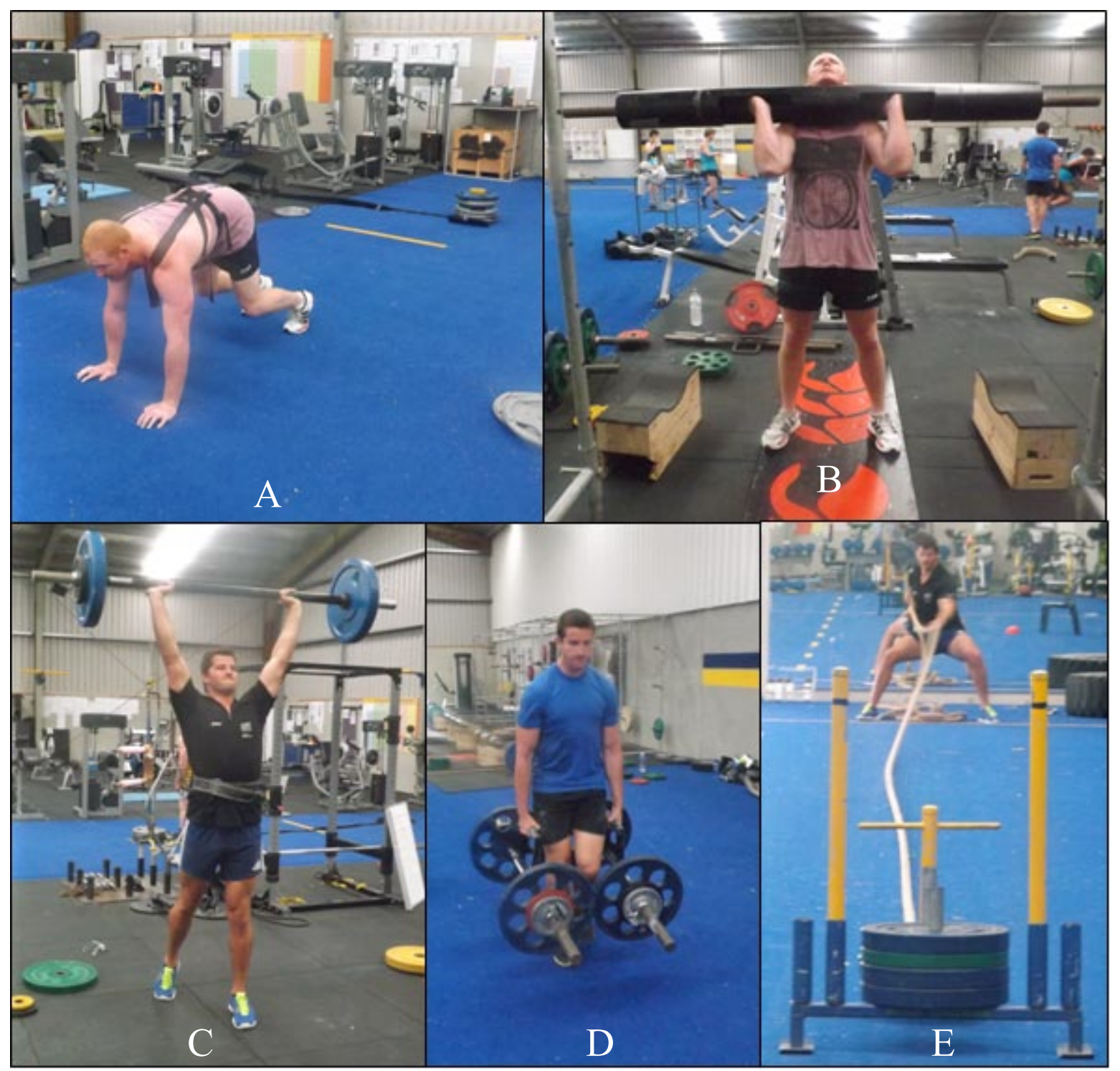

Figure 2: Illustration of various strongman events. $\mathrm{A}=$ Heavy sled pull; $\mathrm{B}=\mathrm{Log}$ lift; $\mathrm{C}=$ Axle press; $\mathrm{D}=$ Farmers walk; $\mathrm{E}=$ Arm over arm prowler pull.

The training programs required the participants to train for up to 75 minutes bi-weekly on non-consecutive days. The training exercises were performed in a controlled manner and loading was increased by $\sim 2 \%$ each week providing the participant could maintain good form. The fourth week was a de-loading week in which participants performed the exercises with the same loads they used in week one. All training sessions were supervised by qualified training instructors and $\operatorname{logs}$ of all participants training sessions were recorded. 
Supplementary training was permitted which consisted of prehabilitation and cardiovascular conditioning. All athletes were encouraged to perform 2 sessions of prehabilition exercises per week and 2 cardiovascular training sessions focused on improving aerobic capacity. However, these forms of training were not able to be monitored by the researchers.

\section{$\underline{\text { Statistical analyses }}$}

The data was explored by a histogram plot, and the normality of distribution was tested using Shapiro-Wilk's test for all groups in this study. Then, descriptive statistics were calculated and reported as mean and standard deviations. The difference in central location (mean) between groups was examined using the independent sample $t$-test. For the data that did not follow a normal distribution, the Mann-Whitney $U$-test was used to determine if the difference between groups was significant. Effect sizes $(\mathrm{ES}=$ mean change/standard deviation of the sample scores) were calculated to quantify the magnitude of the performance differences (i.e. pre intervention results - post intervention results) between each of the two groups (i.e. strongman and traditional) (9). Cohen applied qualitative descriptors for the effect sizes $>0.2,>0.5$ and $>0.8$ indicated small, moderate, and large changes, respectively. To counteract the problem of multiple comparisons and the chance of a false positive, significance was accepted at the $\mathrm{p} \leq 0.01$ level as a compromise between increasing risk of both Type I (finding statistical between-group significance where none truly exists) and Type II (finding no statistical between-group significance where one truly exists) errors. The 95\% confidence interval $(95 \% \mathrm{CI})$ was also calculated for all measures. All statistical analyses were carried out using SPSS 20.0 for Windows (SPSS Inc., Chicago, IL, USA). 


\section{$\underline{\text { Results }}$}

Overall, all strength and functional performance measures tended to improve with training $(0.2 \%$ to $7 \%)$, thus providing evidence that both training programs provided positive training adaptations (see Table 3$)$. However, no significant $(\mathrm{p}<0.01)$ between-group differences were found for the functional performance measures, indicating that there was no statistically significant advantage between traditional and strongman training methods.

With regards to the between group effects traditional training was associated with greater (small-moderate) effect size changes in body fat mass $(\mathrm{ES}=-0.38), \%$ body fat $(\mathrm{ES}=-0.38)$, $1 \mathrm{RM}$ squat $(\mathrm{ES}=0.47)$ and deadlift $(\mathrm{ES}=0.66), \mathrm{COD}$ turning ability $(\mathrm{ES}=-0.38)$ and total COD time $(\mathrm{ES}=-0.25)$, horizontal jump $(\mathrm{ES}=0.56)$, and sled push performance $(\mathrm{ES}=-$ 0.31 to -0.46$)$ than strongman training. Conversely strongman training was found to elicit small-large greater increases in muscle mass $(\mathrm{ES}=0.44), 1 \mathrm{RM}$ bent over row $(\mathrm{ES}=1.10), 5$ $\mathrm{m}(\mathrm{ES}=-0.28)$ sprint performance and $\mathrm{COD}$ acceleration $(\mathrm{ES}=-0.33)$ than traditional training. 
Table 3: Magnitude of differences between pre and post intervention measures tested between traditional and strongman training groups

Between-group differences

\begin{tabular}{|c|c|c|c|c|c|}
\hline & Strongman & Traditional & Difference & $95 \% \mathrm{CI}$ & Effect Size \\
\hline \multicolumn{6}{|l|}{ Body Composition } \\
\hline Body mass (kg) & $0.5 \pm 2.0$ & $-0.5 \pm 2.3$ & $0.0 \pm 0.8$ & -1.6 to 1.6 & 0.00 \\
\hline Muscle mass (kg) & $-0.4 \pm 0.8$ & $-0.0 \pm 1.0$ & $0.4 \pm 0.3$ & -0.3 to 1.1 & $0.44^{\mathrm{S}}$ \\
\hline Body fat mass $(\mathrm{kg})$ & $0.3 \pm 2.0$ & $-0.4 \pm 1.8$ & $-0.7 \pm 0.7$ & -2.2 to 0.8 & $-0.36^{\mathrm{T}}$ \\
\hline Body fat $(\%)$ & $0.3 \pm 2.0$ & $-0.4 \pm 1.6$ & $-0.7 \pm 0.7$ & -2.1 to 0.7 & $-0.38^{\mathrm{T}}$ \\
\hline \multicolumn{6}{|l|}{ IRM Measures } \\
\hline Clean and jerk (kg) & $-7.5 \pm 5.8$ & $-8.7 \pm 6.5$ & $-1.2 \pm 2.6$ & -6.6 to 4.2 & 0.19 \\
\hline Deadlift $(\mathrm{kg})$ & $-10.4 \pm 10.9$ & $-17.8 \pm 11.8$ & $-7.5 \pm 4.7$ & -17.2 to 2.3 & $0.66^{\mathrm{T}}$ \\
\hline Military press $(\mathrm{kg})$ & $-6.2 \pm 6.9$ & $-5.3 \pm 4.8$ & $-0.9 \pm 2.5$ & -4.3 to 6.0 & 0.15 \\
\hline Squat (kg) & $-3.9 \pm 16.1$ & $-10.9 \pm 13.7$ & $-7.0 \pm 6.2$ & -20.0 to 6.0 & $0.47^{\mathrm{T}}$ \\
\hline Bent over row $(\mathrm{kg})$ & $-14.5 \pm 9.0$ & $-4.7 \pm 8.8$ & $9.8 \pm 3.8$ & 1.7 to 17.9 & $1.10^{\mathrm{S}}$ \\
\hline \multicolumn{6}{|l|}{ Functional Performance Measures } \\
\hline \multicolumn{6}{|l|}{ Sprint Speed } \\
\hline $5 \mathrm{~m}(\mathrm{~s})$ & $0.02 \pm 0.04$ & $0.01 \pm 0.03$ & $-0.01 \pm 0.01$ & -0.04 to 0.02 & $-0.28^{\mathrm{S}}$ \\
\hline $15 \mathrm{~m}(\mathrm{~s})$ & $0.01 \pm 0.06$ & $0.01 \pm 0.04$ & $-0.00 \pm 0.02$ & -0.04 to 0.04 & -0.06 \\
\hline $30 \mathrm{~m}(\mathrm{~s})$ & $0.02 \pm 0.10$ & $0.01 \pm 0.06$ & $-0.01 \pm 0.03$ & -0.07 to 0.05 & -0.18 \\
\hline \multicolumn{6}{|l|}{505 COD Test } \\
\hline Deceleration $(-5 \mathrm{~m}$ to $-2 \mathrm{~m})$ (s) & $0.01 \pm 0.03$ & $0.00 \pm 0.04$ & $-0.00 \pm 0.01$ & -0.03 to 0.03 & -0.05 \\
\hline Turning ability $(-2 \mathrm{~m}$ to $2 \mathrm{~m})(\mathrm{s})$ & $0.00 \pm 0.14$ & $0.05 \pm 0.10$ & $0.05 \pm 0.04$ & -0.05 to 0.14 & $-0.38^{\mathrm{T}}$ \\
\hline Acceleration $(2 \mathrm{~m}$ to $5 \mathrm{~m})(\mathrm{s})$ & $0.01 \pm 0.06$ & $-0.02 \pm 0.04$ & $-0.02 \pm 0.02$ & -0.06 to 0.03 & $-0.33^{\mathrm{s}}$ \\
\hline Total time (s) & $0.01 \pm 0.13$ & $0.04 \pm 0.07$ & $0.03 \pm 0.04$ & -0.06 to 0.11 & $-0.25^{\mathrm{T}}$ \\
\hline \multicolumn{6}{|l|}{$15 \mathrm{~m}$ 70kg Sled Push } \\
\hline $5 \mathrm{~m}(\mathrm{~s})$ & $0.02 \pm 0.11$ & $0.09 \pm 0.10$ & $0.07 \pm 0.04$ & -0.02 to 0.15 & $-0.31^{\mathrm{T}}$ \\
\hline $10 \mathrm{~m}(\mathrm{~s})$ & $0.04 \pm 0.18$ & $0.10 \pm 0.14$ & $0.05 \pm 0.06$ & -0.07 to 0.17 & $-0.33^{\mathrm{T}}$ \\
\hline $15 \mathrm{~m}(\mathrm{~s})$ & $0.05 \pm 0.20$ & $0.14 \pm 0.16$ & $0.08 \pm 0.07$ & -0.06 to 0.22 & $-0.46^{\mathrm{T}}$ \\
\hline \multicolumn{6}{|l|}{ Lower body Leg Power } \\
\hline Vertical jump (cm) & $-4.13 \pm 6.35$ & $-3.86 \pm 5.37$ & $-0.28 \pm 2.18$ & -4.20 to 4.75 & 0.09 \\
\hline Horizontal jump (m) & $-0.03 \pm 0.11$ & $-0.09 \pm 0.11$ & $-0.06 \pm 0.04$ & -0.15 to 0.02 & $0.56^{\mathrm{T}}$ \\
\hline \multicolumn{6}{|l|}{ Upper Body Pushing Power } \\
\hline 5kg MB Chest throw (m) & $-0.16 \pm 0.19$ & $-0.15 \pm 0.19$ & $-0.01 \pm 0.07$ & -0.13 to 0.15 & 0.05 \\
\hline \multicolumn{6}{|l|}{ Grip Strength } \\
\hline Grip strength left $(\mathrm{kg})$ & $-3.61 \pm 5.30$ & $-6.57 \pm 7.66$ & $-2.97 \pm 2.43$ & -7.98 to 2.04 & $0.20^{\mathrm{T}}$ \\
\hline Grip strength right (kg) & $-7.27 \pm 6.83$ & $-6.67 \pm 8.69$ & $-0.60 \pm 2.85$ & -5.26 to 6.46 & 0.13 \\
\hline
\end{tabular}

95\% CI (confidence interval) of the difference between measures. Values obtained from subtracting post from pre-testing means.

(S) Training effect towards strongman training, (T) Training effect towards traditional training. 


\section{$\underline{\text { Discussion }}$}

The present study is the first to investigate the effects of a strongman training program versus a traditional training program on a variety of body composition, muscular function and performance measures. This study provided a unique opportunity to compare two forms of resistance training in athletes whose primary training goal was to improve functional performance (strength, power, speed and change of direction) for the sport of rugby union. While both the strongman and traditional training programs produced performance benefits, the principle finding in this study was the non-significant between-group differences in body composition and functional performance measures after seven weeks of resistance training. Thus the hypothesis was primarily rejected as both types of training did not offer a significant advantage over the other for improving these outcomes with a short-term training program.

Small between-group effects to body composition were observed in this study, with the strongman training group having a greater effect in changing muscle mass $(\mathrm{ES}=0.44 ; 1.1 \%$ versus $-0.02 \%$ ). Such results may support the findings of Ghigiarelli and colleagues (12) who suggested that strongman training may be beneficial for improving muscular hypertrophy. Interestingly, small negative effects to body fat mass $(\mathrm{kg})(\mathrm{ES}=-0.36)$ and body fat $(\%)(\mathrm{ES}$ $=-0.38)$ were observed in the traditional training group. Previous researchers $(5,21)$ have suggested that strongman exercises carry very high physiological demands, which may account for the small differences observed in this study.

It appears that bi-weekly supervised progressive strength training, supplemented with prehabilitation and cardiovascular conditioning, was sufficient stimulus to increase maximal strength in experienced resistance-trained athletes. Similar strength improvements were observed between the strongman and traditional groups for the clean and jerk $(8.6 \%$ and 
$10.7 \%)$ and military press ( $9.1 \%$ and $7.6 \%)$ 1RM strength measures; however between-group effect size analyses indicated small $(\mathrm{ES}=0.46: 7.5 \%$ versus $2.7 \%$ ), and moderately greater increases $(\mathrm{ES}=0.66: 11.0 \%$ versus $5.7 \%)$ in squat and deadlift strength, respectively for the traditional than strongman group. Interestingly, a large training effect was observed in the strongman group for the bent over row $(\mathrm{ES}=1.10: 13.6 \%$ versus $4.3 \%)$. The differences in the magnitude of strength improvements between the groups may indicate strength specific adaptations associated with each program. Interestingly, the magnitude of strength improvements are similar to those reported by Argus and colleagues (1) for the bench press (11.1\%) and box squat (11.3\%) in which 33 elite male rugby union players performed five high-volume concurrent strength training sessions per week for 4-weeks. Research has reported enhanced strength improvements with increased frequency of training (18).

The magnitude of traditional 1RM strength improvements seen in the strongman training group were not expected, as the traditional group had a post-strength performance testing advantage as the lifts performed (except the bent over row) were part of the traditional groups program. Researchers have shown that practice of a specific task gives better ability to transfer strength improvements (7). From these results it may be surmised that the strongman exercises utilized in this study have a positive impact on overall strength development.

Improvements in strength and power development can transfer to improved physical capabilities (35). Such results were observed in both training groups with improvements in both upper (seated MB chest press throw $=\sim 0.15 \mathrm{~m}$ ) and lower body (countermovement vertical jump $=\sim 4 \mathrm{~cm}$; horizontal jump $=3$ to $9 \mathrm{~cm}$ ) power measures. Interestingly, the between-group improvements were very similar for the vertical jump and seated MB chest press throw. The similar magnitude of change in functional performance may be due to 
specificity of training. Improvements observed in seated MB chest press throw performance may have been attributed to the upper body pushing action of military and axle press exercises. The clean and jerk and log lift, are mechanically similar to the countermovement vertical jump (involving explosive triple extension that occurs at the ankle, knee and hip) and the motor unit firing patterns that are improved during the training of these exercises would likely enhance the firing pattern of these motor units during the countermovement vertical jump as well (34). Researchers utilising weightlifting, kettlebell training and vertical jumping exercises have reported significant improvements $(1-7 \%)$ in vertical jump performance $(28,37)$.

An interesting between-group finding in the present study was that the traditional group demonstrated a greater training effect in horizontal jump performance $(\mathrm{ES}=0.56)$ than the strongman training group. The greater moderate improvement in horizontal jump performance $(3.8 \%$ versus $1.3 \%)$ may have been attributed to the greater strength improvements seen in the squat and deadlift which are performed bilaterally.

In contrast, the strongman training group performed heavy sled pulls and farmers walks which involved periods of unilateral and bilateral work and the production of vertical and horizontal propulsive impulses. Interestingly, the strongman training between-group effects were greater for the $5 \mathrm{~m}(\mathrm{ES}=-0.28 ; 1.8 \%$ faster versus $0.9 \%$ faster $)$ and acceleration phase of the $5-0-5$ COD test $(E S=-0.33 ; 1.5 \%$ faster versus $3 \%$ slower). While the effects are only small, improvements in initial acceleration are important training effects for rugby players as they may provide the player sufficient power to break through tackles and make territorial gains in a match situation. Researchers have reported that heavy sled pulls $(33.1 \pm 5.9 \mathrm{~kg})$ are a sufficient training stimulus to improve both 5 and $10 \mathrm{~m}$ sprint times (19) and are commonly 
used by coaches in strength and conditioning practice (42). The results of this study may support the tenet that specific functional performance adaptation is closely related to the resisted movement patterns associated with the strength and conditioning stimulus. Longerterm training studies could allow better insight into the effectiveness of the heavy sled pull as a conditioning method in improving acceleration performance.

The small differences between the pre-and-post measures for $30 \mathrm{~m}$ speed and change of direction times $(0.2 \%$ to $1.7 \%)$ are consistent with other short-term training studies that have examined the effect of two different resistance-training programs $(17,23,37)$. The results of these studies would indicate that various resistance-training modalities could produce moderate gains in strength and power but only modest changes in speed and COD times. Combinations of high force and high velocity training could result in adaptation occurring at differential parts of the force-velocity curve and therefore have greater impact on athletic performance $(14,17,41,45)$.

A surprising finding for the traditional training group in this study was the training effects $(\sim 3.4 \%$ versus $\sim 1.2 \%)$ associated with the $70 \mathrm{~kg}$ sled push $(5 \mathrm{~m}, \mathrm{ES}=-0.31 ; 10 \mathrm{~m}, \mathrm{ES}=-$ $0.33 ; 15 \mathrm{~m}, \mathrm{ES}=-0.46)$. Effects in favour of the traditional style training were not expected as it was thought that the strongman group would improve sled push performance substantially, given that this group had performed the heavy sled pull for seven weeks as part of the strongman training program. The findings may indicate that the sled pull and sled push elicit different physiological adaptations, or it could be that the strength adaptations associated with the traditional lifts (i.e. squat and deadlift), have better transferability to the horizontal activities (such as the sled push and horizontal jump), a result somewhat counterintuitive (31). Recent research has demonstrated that deep squat $\left(0-120^{\circ}\right.$ of knee 
flexion) training (with loads of 5-10 RM) resulted in greater increases in front thigh muscle CSA, isometric knee extension strength (at $75^{\circ}$ and $105^{\circ}$ knee extension) and squat jump performance than 12 weeks of shallow squat training (with loads of $5-10 \mathrm{RM}$ ) (6). The strongman exercises seen in this study (e.g. farmers walk and sled pull) are performed with less knee and hip flexion than those seen in the squat and deadlift. Such differences may give insight into the small to moderate effects favouring the traditional group in some of the performance measures seen in the present study.

Previous researchers have reported significant increases in grip strength $(5 \%-7 \%)$ among rugby players after 12 weeks of resistance training (36). Both training groups in this study improved grip strength performance (5 to $13 \%$ ). It was thought that the strongman training group would show a much greater improvement than the traditional group as the strongman training implements, such as the axle and farmers bars, were thicker than the Olympic bars used in the traditional group. The thicker bars associated with strongman implements have the potential of enhancing grip strength because of the higher degree of difficulty performing exercises while grasping the bar in an area of range of motion where gripping ability is relatively weak $(8,32)$. A limitation to this study was that grip strength was measured with a handgrip strength dynamometer at one angle (which was similar to the thickness of an Olympic bar). Future investigations could test grip strength at different angles, which may give better insight into the grip strength adaptations associated with training implements of varying widths.

A review by Zemke and colleagues (46) suggested that strongman training programs could help increase adherence to resistance training programs. The results of this study found that 
adherence to training was the same for both groups $(98.6 \%)$; however the strength and conditioning coaches who oversaw the training in both groups were diligent in monitoring the athletes who participated in this study. Future research may wish to consider giving athletes a self-directed approach to training, which may give a better indication of motivation and program adherence.

Research on the injury epidemiology of strongman athletes found that strongman implement training carried twice the risk of injury as traditional training methods (43). While two injuries were reported in this training study, the athlete who had the shoulder injury associated with the strongman program pulled out of the study. Strength and conditioning coaches who utilize strongman training methods should take into consideration the increased risk of potential injury and follow structured conditioning programs with a periodized approach. Such an approach would help to ensure appropriate loading strategies for training phases and planned exercise progressions to ensure technical competency with these lifts/events.

The present study sought to collect data from a number of performance tests to gain greater insight into many aspects of muscular function and performance influenced by the training programs. However, such in-depth analysis is problematic with the issues of statistical significance. The uses of effect sizes were particularly useful for comparing the relative sizes of effects between the different programs and may better demonstrate 'practical significance', particularly if a longer period of training was performed and its effects quantified. Such an approach may be warranted in studies using experienced resistance trained athletes in which increase in performance measures may only be marginal. 
In conclusion, this study compared the short term effects of strongman training and traditional training programs on aspects of muscular function and performance. While the between group effects demonstrated that each program may have advantages in eliciting specific performance gains, no significant between-group differences were found for the functional performance measures. It seems that when exercises are similar and, load and time under tension are equated, short-term strongman training programs are as effective as traditional training programs in improving aspects of muscular function and performance.

\section{Practical Applications}

This study was the first to compare the magnitude of performance changes between a strongman and traditional training program. From a practical perspective, these findings provide conditioning coaches with the first evidence of the efficacy of strongman-training exercises, which can be used by coaches to improve training practices. From the results of this study it can be concluded that strongman training exercises should be considered as possible alternatives to help supplement traditional training approaches. Strongman exercises could offer variation and help improve athlete motivation. Future training studies should investigate the long-term chronic adaptations associated with each strongman implement and the effectiveness of a combined strongman/traditional program versus a traditional program. Such studies would build on the findings of this research and provide practitioners with an evidence base on the performance adaptations associated with strongman implements. This in turn would help improve knowledge regarding the possible utilization of strongman exercises in traditional training programs to further maximise performance enhancements. 


\section{$\underline{\text { References }}$}

1. Argus, CK, Gill, N, Keogh, J, Hopkins, WG, and Beaven, M. Effects of a short-term pre-season training programme on the body composition and anaerobic performance of professional rugby union players. Journal of Sports Sciences. 28:679-686, 2010.

2. Baker, D. Strongman training for large groups of athletes. Journal of Australian Strength and Conditioning. 16:33, 2008.

3. Baker, D and Nance, S. The relation between strength and power in professional rugby league players. Journal of Strength and Conditioning Research. 13:224-229, 1999.

4. Bennett, S. Using "strongman" exercises in training. Strength and Conditioning Journal. 30:42-43, 2008.

5. Berning, JM, Adams, KJ, Climstein, M, and Stamford, BA. Metabolic demands of "junkyard" training: Pushing and pulling a motor vehicle. Journal of Strength and Conditioning Research. 21:853-856, 2007.

6. Bloomquist, K, Langberg, H, Karlsen, S, Madsgaard, S, Boesen, M, and Raastad, T. Effect of range of motion in heavy load squatting on muscle and tendon adaptations. European Journal of Applied Physiology. 113:2133-2142, 2013.

7. Bobbert, EW and Van Soest, AJ. Effects of muscle strengthening on vertical jump height: a simulation study. Medicine \& Science in Sports \& Exercise. 26:1012-1020, 1994.

8. Channel, S. The fat bar. National Strength and Conditioning Association Journal. 12:26-27, 1990.

9. Cohen, J. Statistical power analysis for the behavioural science. Hillside, New Jersey: Lawrence Erlbaum Associates, 1988.

10. Corcoran, G and Bird, S. Preseason strength training for rugby union: The general and specific preparatory phases. Strength and Conditioning Journal. 31:66-74, 2009.

11. Gabbett, T, Kelly, JN, and Sheppard, JM. Speed, change of direction speed, and reactive agility of rugby league players. Journal of Strength \& Conditioning Research. 22:174-181, 2008.

12. Ghigiarelli, J, Sell, K, Raddock, J, and Taveras, K. The effects of strongman training on salivary testosterone levels in a sample group of trained males Journal of Strength \& Conditioning Research. 27:738-747, 2013. 
13. Harasin, D, Dizdar, D, and Markovic, G. High reliability of tests of maximum throwing performance. Journal of Human Movement Studies. 51:63-76, 2006.

14. Harris, GR, Stone, MH, O’Bryant, HS, Proulx, CM, and Johnson, RL. Short-term performance effects of high power, high force, or combined weight-training methods. Journal of Strength \& Conditioning Research. 14:14-20, 2000.

15. Hedrick, A. Training for high-performance collegiate ice hockey. Strength and Conditioning Journal. 24:42-52, 2002.

16. Hedrick, A. Using uncommon implements in the training programs of athletes. Strength and Conditioning Journal. 25:18-22, 2003.

17. Hoffman, JR, Cooper, J, Wendell, M, and Kang, J. Comparison of Olympic vs. traditional power lifting training programs in football players. Journal of Strength \& Conditioning Research. 18:129-135, 2004.

18. Hoffman, JR, Kraemer, WJ, Fry, AC, Deschenes, M, and Kemp, M. The effects of self-selection for frequency of training in a winter conditioning program for football. Journal of Applied Sport Science Research. 4:76-82, 1990.

19. Kawamori, N, Newton, RU, Hori, N, and Nosaka, K. Effects of weighted sled towing with heavy versus light load on sprint acceleration ability. Journal of Strength \& Conditioning Research In Press.

20. Keogh, J. Lower body resistance training: Improving functional performance with lunges. Strength and Conditioning Journal. 21:67-72, 1999.

21. Keogh, JWL, Newlands, C, Blewett, S, Payne, A, and Chun-Er, L. A kinematic analysis of a strongman event: The heavy sprint-style sled pull. Journal of Strength and Conditioning Research. 24:3088-3097, 2010.

22. Keogh, JWL, Payne, AL, Anderson, BB, and Atkins, PJ. A brief description of the biomechanics and physiology of a strongman event: The tire flip. Journal of Strength and Conditioning Research. 24:1223-1228, 2010.

23. Kibele, A and Behm, DG. Seven weeks of of instability and traditional resistance training effects of strength, balance and functional performance. Journal of Strength \& Conditioning Research. 23:2443-2450, 2009.

24. Linthorne, NP and Cooper, JE. Effect of the coefficient of friction of a running surface on sprint time in a sled-towing exercise. Sports Biomechanics. 12:175-185, 2013. 
25. Maulder, $\mathrm{P}$ and Cronin, J. Horizontal and vertical jump assessment: reliability, symmetry, discriminative and predictive ability. Physical Therapy in Sport. 6:74-82, 2005.

26. McGill, SM, McDermott, A, and Fenwick, CMJ. Comparison of different strongman events: Trunk muscle activation and lumbar spine motion, load, and stiffness. Journal of Strength and Conditioning Research. 23:1148-1161, 2009.

27. Moir, G, Button, C, Glaister, M, and Stone, MH. Influence of familiarization on the reliability of vertical jump and acceleration sprinting performance in physically active men. Journal of Strength \& Conditioning Research. 18:276-280, 2004.

28. Otto, WH, Coburn, JW, Brown, LE, and Spiering, BA. Effects of weightlifting vs. kettlebell training on vertical jump, strength, and body composition. Journal of Strength \& Conditioning Research. 26:1199-1202, 2012.

29. Poliquin, C. The Poliquin Principles: Successful Methods for Strength and Mass Development Napa Valley, California: Dayton Writers Group, 1997.

30. Poliquin, $\mathrm{C}$ and McDermott, A. Applied strongman training for sport - theory and technical. Nevada City, CA: Ironmind Enterprizes Inc, 2005.

31. Randell, AD, Cronin, JB, Keogh, JWL, and Gill, ND. Transference of strength and power adaptation to sports performance - horizontal and vertical force production. Strength and Conditioning Journal. 32:100-106, 2010.

32. Ratamess, NA, Faigenbaum, AD, Mangine, GT, Hoffman, JR, and King, J. Acute muscular strength assessment using free weight bars of different thickness Journal of Strength and Conditioning Research. 21:240-244, 2007.

33. Stone, M, Stone, M, and Sands, AW. Modes of resistance training. In: Principles and practice of resistance training. ed. Champaign, IL: Human Kinetics, 2007. pp. 241257.

34. Stone, MH, Byrd, R, Tew, J, and Wood, M. Relationship between anaerobic power and olympic weightlifting performance. Journal of Sports Medicine and Physical Fitness. 20:99-102, 1980.

35. Stone, MH, Moir, G, Glaister, M, and Sanders, R. How much strength is necessary? Physical Therapy in Sport. 3:88-96, 2002.

36. Tong, RJ and Mayes, R, The effect of pre-season training on the physiological characteristic of international rugby players, in Science and Football III: 
Proceedings of the Third World Congress of Science and Football, Reilly T, Bangsbo J, and Hughes M, Editors. 1995: Cardiff, Wales.

37. Tricoli, V, Lamas, L, Carnevale, R, and Ugrinowitsch, C. Short-term effects on lowerbody functional power development: Weightlifting vs. vertical jump training programmes. Journal of Strength and Conditioning Research. 19:433-437, 2005.

38. Waller, M, Piper, T, and Townsend, R. Strongman events and strength and conditioning programs. Strength and Conditioning Journal. 25:44-52, 2003.

39. Wang, CY and Chen, LY. Grip strength in older adults: test-retest reliability and cutoff for subjective weakness of using the hands in heavy tasks. Archives of Physical Medicine and Rehabilitation. 91:1747-1751, 2010.

40. Wilson, G. Strength and power assessment. In: Applied Anatomy and Biomechanics in Sport. ed. Melbourne: Blackwell Scientific Publications, 1994.

41. Wilson, GJ, Newton, RU, Murphy, AJ, and Humphries, BJ. The optimal training load for the development of dynamic athletic performance. Medicine \& Science in Sports \& Exercise. 25:1279-1286, 1993.

42. Winwood, PW, Cronin, JB, Dudson, MK, Gill, ND, and Keogh, JWL. How coaches use strongman implements in strength and conditioning practice. International Journal of Sports Science \& Coaching In Press.

43. Winwood, PW, Hume, PA, Cronin, JB, and Keogh, JWL. Retrospective injury epidemiology of strongman athletes. Journal of Strength \& Conditioning Research. 28:28-42, 2014.

44. Winwood, PW, Keogh, JWL, and Harris, NK. The strength and conditioning practices of strongman competitors. Journal of Strength and Conditioning Research. 25:31183128, 2011.

45. Zafeiridis, A, Saraslandis, P, Manou, V, Ioakimidis, P, Dipla, K, and Kellis, S. The effects of resisted sled-pulling sprint training on acceleration and maximum speed performance. Journal of Sports Medicine and Physical Fitness. 45:284-290, 2005.

46. Zemke, B and Wright, G. The use of strongman type implements and training to increase sport performance in collegiate athletes. Strength and Conditioning Journal. 33:1-7, 2011. 


\section{Appendix 1: TECHNICAL NOTE}

\section{Matching Loading Parameters for the Strongman Events and Traditional Exercises}

Six male strongman athletes (four national and two local level athletes) volunteered to participate in the biomechanical analysis (mean \pm SD: age $24.0 \pm 3.9 \mathrm{yr}$; stature $181.6 \pm 9.4 \mathrm{~cm}$; body mass 112.9 $\pm 28.9 \mathrm{~kg}$ ). Data were collected for each participant over 2 sessions separated by 1 week. Session 1 was performed in the strength and conditioning laboratory and involved 1-repetition maximum $(1 \mathrm{RM})$ testing in the squat, deadlift and clean and jerk. Session 2 was performed in the biomechanics laboratory where participants performed repetitions in the squat, deadlift, clean and jerk, farmers walk, log lift and heavy sled pull using the traditional lift (equivalent) loads of $70 \% 1 \mathrm{RM}$. Kinetics were analysed during session 2 only.

A Bertec force plate (Model AM6501, Bertec Corp., Columbus, OH, USA) was used to collect synchronized ground reaction forces at $1000 \mathrm{~Hz}$. Vicon Nexus (Version 1.8.1, Vicon Inc., Denver, CO, USA) was used to process the ground reaction force data. Ground reaction force data were filtered using a fourth order low-pass digital Butterworth filter with a cut-off frequency of $6 \mathrm{~Hz}$.

To calculate and match exercise loading parameters resultant forces were calculated using; square $\operatorname{root}\left(\mathrm{X}^{2}+\mathrm{Y}^{2}+\mathrm{Z}^{2}\right)$ 
Table 4: Calculations of Resultant Forces

\begin{tabular}{|c|c|}
\hline Dea & Far \\
\hline $\begin{array}{l}\text { Total Resultant Forces: } \sqrt{ }\left(\mathrm{X}^{2}+\mathrm{Y}^{2}+\mathrm{Z}^{2}\right) \\
=\text { Square root }\left(0.15^{2}+3.50^{2}+2688.32^{2}\right) \\
=\text { Square root }(0.023+12.25+7225344.4) \\
=2688.00 \mathrm{~N} \\
\text { Total lift time }=3.95 \text { seconds }\end{array}$ & $\begin{array}{l}\text { Total Resultant Forces: } \sqrt{ }\left(\mathrm{X}^{2}+\mathrm{Y}^{2}+\mathrm{Z}^{2}\right) \\
\text { Square root/ }\left(13.05^{2}+-31.50^{2}+2532.72^{2}\right) \\
=\text { Square root } /(182.25+22153.35+6414670.5) \\
=2535.12 \mathrm{~N} \\
\text { Average velocity }=1.48 \mathrm{~m} / \mathrm{s}\end{array}$ \\
\hline \multicolumn{2}{|c|}{$\begin{array}{l}\text { No significant differences were found in the sum of resultant mean forces between the farmers } \\
\text { walk }(3-4 \mathrm{~m}) \text { and deadlift. Loading was equated by time under tension. One full deadlift } \\
\text { repetition (i.e. concentric \& eccentric phases) with a } 70 \% 1 \mathrm{RM} \text { load, took } 3.95 \text { seconds which } \\
\text { equated to a distance of } 5.85 \mathrm{~m} \text { in the farmers walk with a load of } 70 \% 1 \mathrm{RM} \text { deadlift. The } \\
\text { initial lift of the farmers lift }(0.92 \mathrm{sec}) \text { will take } 1 \mathrm{~m} \text { off total distance calculated. } \\
\text { Therefore: } 5^{*} \text { Deadlift reps }=28 \mathrm{~m} \text { of farmers walking with the same given load. }\end{array}$} \\
\hline $\mathrm{S}$ & \\
\hline $\begin{array}{l}\text { Total Resultant Forces: } \sqrt{ }\left(\mathrm{X}^{2}+\mathrm{Y}^{2}+\mathrm{Z}^{2}\right) \\
=\text { Square root }\left(-3.23^{2}+-7.78^{2}+2579.22^{2}\right) \\
=\text { Square root }(10.4+60.5+6652375.8) \\
=2579.2 \mathrm{~N} \\
\text { Total lift time }=2.81 \text { seconds }\end{array}$ & $\begin{array}{l}\text { Total Resultant Forces: } \sqrt{ }\left(\mathrm{X}^{2}+\mathrm{Y}^{2}+\mathrm{Z}^{2}\right) \\
\text { Square root } /\left(-5.45^{2}+270.82^{2}+1268.95^{2}\right) \\
=\text { Square } \operatorname{root} /(29.7+73343.5+1610234.1) \\
=1297.5 \mathrm{~N} \\
\text { Average velocity }=1.83 \mathrm{~m} / \mathrm{s} \\
\text { Step length } 0.645 \mathrm{~m} \\
\text { Stride length } 1.29 \mathrm{~m}\end{array}$ \\
\hline \multicolumn{2}{|c|}{$\begin{array}{l}\text { Significant differences were found in the sum of mean resultant forces between the squat and } \\
\text { sled pull mean forces. The resultant force for the squat was } 2579.2 \mathrm{~N} \text { which was twice the } \\
\text { magnitude of one stride in the sled pull }(1297.5 \mathrm{~N}) \text { (difference between bilateral versus } \\
\text { unilateral). Loading was equated by time under tension. One full squat repetition (i.e. } \\
\text { concentric \& eccentric phases) with a } 70 \% 1 \mathrm{RM} \text { load, took } 2.81 \text { seconds which equated to a } \\
\text { distance of } 5.14 \mathrm{~m} \text { in the sled pull with a load of } 70 \% 1 \mathrm{RM} \text { squat. } \\
\text { Therefore: } 5^{*} \text { squat reps }=25 \mathrm{~m} \text { of sled pulling with the same given load. } \\
\text { Note: } 0.7 \mathrm{~m} \text { taken off total sled pull distance to accommodate co-efficient of friction } \\
(0.21 \pm 0.01 \mu)(24) \text {. }\end{array}$} \\
\hline
\end{tabular}




\begin{tabular}{|c|c|}
\hline Clean and Jerk & Log Lift \\
\hline $\begin{array}{l}\text { Total Resultant Forces: } \sqrt{ }\left(\mathrm{X}^{2}+\mathrm{Y}^{2}+\mathrm{Z}^{2}\right) \\
=\text { Square } \operatorname{root}\left(2.36^{2}+2.01^{2}+1921.47^{2}\right) \\
=\text { Square } \operatorname{root}(5.57+4.04+3692046.9) \\
=1921.5 \mathrm{~N} \\
\text { Total lift time }=6.20 \text { seconds }\end{array}$ & $\begin{array}{l}\text { Total Resultant Forces: } \sqrt{ }\left(\mathrm{X}^{2}+\mathrm{Y}^{2}+\mathrm{Z}^{2}\right) \\
\text { Square root/ }\left(2.12^{2}+0.86^{2}+1940.26^{2}\right) \\
=\text { Square root } /(4.5+0.74+3764608.9) \\
=1940.3 \mathrm{~N} \\
\text { Total lift time }=7.96 \text { seconds }\end{array}$ \\
\hline \multicolumn{2}{|c|}{$\begin{array}{l}\text { No significant differences were observed in lift times and sum of resultant forces. Therefore } \\
\text { training loads and reps were equal between the clean and jerk and log lift. } \\
5^{*} \text { Clean and Jerks reps }=5^{*} \log \text { Lifts }\end{array}$} \\
\hline
\end{tabular}

